\title{
Unraveling the Influence of the Interplay Between Mobile Phones' and Users' Awareness on the User Experience (UX) of Using Mobile Phones
}

\author{
Qin, Xiangang; Tan, Chee-Wee; Clemmensen, Torkil
}

Document Version

Accepted author manuscript

Published in:

Human Work Interaction Design. Designing Engaging Automation

DOI:

10.1007/978-3-030-05297-3_5

Publication date:

2019

License

Unspecified

Citation for published version (APA):

Qin, X., Tan, C-W., \& Clemmensen, T. (2019). Unraveling the Influence of the Interplay Between Mobile Phones' and Users' Awareness on the User Experience (UX) of Using Mobile Phones. In B. R. Barricelli, V. Roto, T. Clemmensen, P. Campos, A. Lopes, F. Gonçalves, \& J. Abdelnour-Nocera (Eds.), Human Work Interaction Design. Designing Engaging Automation: Revised Selected Papers of the 5th IFIP WG 13.6 Working Conference. HWID 2018 (pp. 69-84). Springer. IFIP Advances in Information and Communication Technology Vol. 544 https://doi.org/10.1007/978-3-030-05297-3_5

Link to publication in CBS Research Portal

\section{General rights}

Copyright and moral rights for the publications made accessible in the public portal are retained by the authors and/or other copyright owners and it is a condition of accessing publications that users recognise and abide by the legal requirements associated with these rights.

\section{Take down policy}

If you believe that this document breaches copyright please contact us (research.lib@cbs.dk) providing details, and we will remove access to the work immediately and investigate your claim. 


\section{Unraveling the Influence of the Interplay Between Mobile Phones' and Users' Awareness on the User Experience (UX) of Using Mobile Phones Kiangang Qin, Chee-Wee Tan, and Torkil Clemmensen}

Article in proceedings (Accepted version*)

\section{Please cite this article as:}

Qin, X., Tan, 〔-W., \& Clemmensen, T. (2019). Unraveling the Influence of the Interplay Between Mobile Phones' and Users' Awareness on the User Experience (UX) of Using Mobile Phones. In B. R. Barricelli, V. Roto, T. Clemmensen, P. Campos, A. Lopes, F. Gonçalves, \& J. Abdelnour-Nocera (Eds.), Human Work Interaction Design. Designing Engaging Automation: Revised Selected Papers of the 5th IFIP WG 13.6 Working Conference. HWID 2018 (pp. 69-84). Cham: Springer. IFIP Advances in Information and Communication Technology, Vol.. 544, 001: 10.1007/978-3-030-05297-3_5

This is a post-peer-review, pre-copyedit version of an article published in Human Work Interaction Design. Designing Engaging Automation: Revised Selected Papers of the 5th IFIP WG 13.6 Working Conference. HWID 2018. The final authenticated version is available online at:

DOl: https://doi.org/10.1007/978-3-030-05297-3_5

* This version of the article has been accepted for publication and undergone full peer review but has not been through the copyediting, typesetting, pagination and proofreading process, which may lead to differences between this version and the publisher's final version AKA Version of Record.

Uploaded to CBS Research Portal: February 2019 


\title{
Unraveling the Influence of the Interplay between Smart Phones' and Users' Awareness on the User Experience (UX) of Mobile Context-Aware Applications
}

\author{
Xiangang Qin ${ }^{1,2}$, Chee-Wee Tan ${ }^{1}$ and Torkil Clemmensen ${ }^{1}$ \\ ${ }^{1}$ Copenhagen Business School, Denmark \\ ${ }^{2}$ Beijing University of Posts \& Telecommunications, China \\ \{tc.digi,ct.digi,xq.digi\}@cbs.dk
}

\begin{abstract}
The effect imposed by the interplay between smart phones' and users' awareness on the User Experience (UX) of mobile context-aware applications remains unclear. To bridge the knowledge gap, a week-long logging study with 32 participants together with a follow-up survey were conducted. We discovered that the usage of smart phone applications are not only initiated by the selfawareness of users, but they are also dependent on contextual factors. Active awareness of smart phones deteriorates easily due to decreasing computing resources caused by increasing usage. This in turn triggers passive awareness in users. We advance a conceptual model and discuss the implications for designing mobile context-aware applications. Findings from this study lay the groundwork for comprehending the dynamics of UX for mobile context-aware applications.
\end{abstract}

Keywords: Mobile Context Awareness; User Experience; Smart Phone Usage.

\section{$1 \quad$ Introduction}

Active mobile devices have by now outnumbered the global population with the vast majority being smart phones [1]. Indeed, smart phones have permeated every aspect of our daily life in that their usage has become almost indispensable for both personal and professional activities. Although it is indisputable that smart phones could enhance job performance, their usage at work may also distract users from concentrating on tasks at hand, thereby leading to reduced productivity [2-4]. In fact, the situation is worsening given that: (a) smart phones are carried and utilized by individuals anywhere and anytime; (b) their usage is subjected to diverse physical and social contexts; (c) the amount of contextual information being captured by smart phone services is growing, and; (d) smart phone users are living in an increasingly active information environment $[5,6]$. Because it is both tedious and time-consuming for individual to manually monitor and react to ever-changing contexts (e.g., surrounding light conditions), context-aware services could aid smart phone users to be vigilant of changes in their contextual environment by mitigating challenges with information overload $[5,7,8]$. 
Even though smart phones are capable of capturing contextual information, studies have advocated that the quality of User Experience (UX) for mobile context-aware applications is dependent whether these applications embody two essential properties: (a) predicting users' intention and executing actions automatically without evoking users' awareness, as well as; (b) involving varied levels of user awareness in the loop instead of taking awareness away from users [9-12]. In the field of automation, numerous studies have alluded to human performance problems (e.g., complacency, loss of situational awareness, and vigilance deprovement) in complex, automated systems that are caused by excluding humans from the loop [13, 14]. Specifically, performance problems in automated systems may be caused by over-reliance on automation, humans' role as passive monitor rather than active processor of contextual information when interacting with automated systems as well as the absence of feedback from automated systems. We therefore posit that delivering the passive awareness of contextual information (e.g., notifications) while safeguarding the active awareness of smart phone users, is critical to the success of mobile context-aware applications. In turn, the interplay between smart phones' and users' awareness constitutes a Hybrid Awareness System (HAS) that dictates the behavior of humancomputer interaction systems as a collective $[11,12,15]$.

This study conceives awareness as a property of both smart phones and their users. In this sense, we contribute to the working conference on Human Work Interaction Design by connecting the analysis of human work, learning, and leisure activities with the design of the mobile interaction [16-18]. Our conceptualization of awareness hence departs from contemporary definitions that are tailored specifically for other fields of study. For example, in the field of Computer-Supported Cooperative Work, awareness has been construed, from the human perspective, as "the amount of knowledge that a person has about a topic in particular" [19]. Conversely, within the technically-oriented context-aware community, awareness has been defined as "the ability of the system to leverage the context to provide the appropriate response to the users"[12]. In contrast, our HAS approach strives for the middle ground by theorizing awareness as the encapsulation of both smart phones' and users' awareness. For this reason, HAS should entail: (1) sensing ability for acquiring contextual information (i.e., computing, physical, social, and user contexts) to be utilized subsequently by smart phones or users to anticipate and decide on appropriate actions; (2) comprehension capability for interpreting the significance of acquired information, as well as; (3) execution capacity for acting appropriately in accordance with interpreted information.

A good UX of HAS demands seamless collaboration between smart phones' and users' awareness by striking a balance between active and passive awareness for both enitites. While context-awareness promises to diminish users' mental workload by improving the usability of smart phones and rendering users' interactions with these devices to be more efficient or less effortful [20], the broad range of contextual-aware services afforded by smart phones could still culminate in issues like information overload, loss of control, mental distress, privacy violation, untimely distraction and unwanted interruption [21,22]. Despite expending tremendous effort to bolster passive awareness by pushing notifications to smart phone users during opportune moments [23-25], studies have shown that users tend to engage in proactive 'checking habits' by 
performing brief and repeated inspection of dynamic content, which can be quickly accessed from the device [7]. Certain users have even developed obsessive-compulsive inclinations by being actively aware of their smart phones to the extent that they interrupt their ongoing tasks to inspect dynamic content on these devices even when notifications were turned off $[8,26,27]$. We thus postulate that users should retain a reasonable degree of active self-awareness such that context-aware services complement and extend users' awareness only when the latter is inadequate. In other words, context-aware services should not supplant users' awareness by being overly sensitive to contextual information or even, at times, wrestling control away from users [9].

In spite of extensive research into context-aware services (i.e., conceptual algorithms, network infrastructure, middleware, and applications), little attention has been paid to the interplay between smart phones and users in being aware of diverse contexts from a human-centric perspective $[9,11,21,28,29]$. Consequently, we collected data on (a) 'Turning Screen On' events to examine the Human ActiveComputer Passive mode; (b) 'Light change' and 'Auto-brightness' events to explore the Computer Active-Human Passive mode, as well as; (c) conducted surveys to ascertain users' subjective assessment of select context-aware services. Building on our earlier work [30][31], where we put forth a preliminary framework to explicate the UX of mobile context-aware applications from users' perspective based on focus group discussions, this study embraces a mixed-methods approach to unravel UX considerations related to such services [32]. In this way, findings from our study contribute to an in-depth appreciation of how UX is shaped by the interplay in between smart phones' and users' awareness, which in turn can inform the design of mobile context-aware applications.

\section{Methodology}

\subsection{Method}

Data was collected via a mixed method approach before employing the Grounded Theory Method (GTM) to analyze the data and construct the conceptual model for this study. A log study and a diary study, along with post-hoc semi-structured interviews, were conducted to unravel the interplay between smart phones' and users' awareness. Post-hoc interviews and open-ended questions from the diary study were analyzed with an axial coding strategy [33] to derive the causal conditions underlying the interplay between smart phones' and users' awareness, the strategies for managing this interplay in awareness, as well as the consequence of the interplay on UX.

\subsection{Participants}

The study was conducted with 15 Danish and 17 Chinese Android Phone users, ranging from 19 to 28 years of age. Participants were students with 15 out of 17 Chinese participants belonging to a design department of a technical university in Beijing, and 
the remaining 15 Danish participants from a variety of higher education studies including design, law, and medicine. All participants had owned an Android smart phone for no less than half a year. Each participant was compensated with $350 \mathrm{DKK}$ or RMB for their participation. We developed a data harvesting tool based on the Android studio software that allows us to capture contextual information with open APIs.

\subsection{Research Phases}

Prior to consent, participants were notified of the purpose of the study and the types of data to be collected. The entire research lasted for approximately four months and was carried out in four phases consecutively.

First Phase: Collecting log data of usage behavior about application, sensor data of contextual events and diary data of UX with two tailored tools (MOCCA.Capture and MOCCA.Diary) developed for this study. This phase lasted for a week for each participant.

\begin{tabular}{c|c}
\hline Data Tag & Time Stamps \\
\hline com.sina.weibo & $8: 00: 27$ \\
\hline Low light level & $8: 21: 23$ \\
\hline
\end{tabular}

Table 1. Sample of Log Data

Second Phase: Analysis of usage behavior, contextual events and diary data. The sensor and log data was analyzed individually by a tool developed with MS function to illuminate the frequency of application usage, physical contextual events and computing contextual events that transpired during the 7 days in the first phase. Secondly, participants documented their usage experience with 30 separate contextaware services that had been sampled from the mobile applications running on Android mobile phones, rated their satisfaction in interacting with these services, and articulated the rationale behind their rating.

Third Phase: Questionnaire survey and post-hoc interviews were conducted to decipher the phenomenon unveiled by user behavior, contextual event, and diary study. Participants were asked to rate their feeling of 30 context-aware services if they have utilized one or more of these services before.

Fourth Phase: Analysis of survey data. GTM [33] was employed to guide the coding of raw survey data to construct our conceptual model. Interview transcripts were also revisited to discover the interactional strategies deployed by users to manage and handle the dynamism in interplay between smart phones' and users' awareness. Finally, the impact of this interplay on UX was analyzed. 


\section{Results}

\subsection{Active Human and Passive Computer Awareness}

Turning the screen on by a user represents a typical type of interaction event initiated through active user awareness (human awareness). We analyzed the relationship between preceding and follow-up usage sessions of the 'Turning Screen On' event with the aim of uncovering how the interaction initiated by active user awareness disturbed the ongoing status of users. Results indicate that 519 (49.7\%) of the 1046 usage sessions following Turning screen on is 'Turning screen off', 52\% of the time from Turning screen on to Turning screen off is 5-15s (Fig.2), followed by less than 5s (19\%), 15-30s $(11 \%)$, longer than 70 s $(10 \%)$ and $30-70$ s $(8 \%)$. By comparison, $36 \%$ of the other 526 $(50.3 \%)$ events, which transpired after Turning screen on, were completed within $5 \mathrm{~s}$, followed by $5-15 \mathrm{~s}(34 \%), 30-70 \mathrm{~s}(14 \%), 15-30 \mathrm{~s}(9 \%)$ and longer than $70 \mathrm{~s}$ $(8 \%)(\chi 2=11.56, p<.05)$. These results imply that users, after turning on their smart phones and checking the content for $15 \mathrm{~s}$, display awareness in turning off the screen for around $35 \%$ of the situations. Further analysis of the preceding and follow-up usage sessions (104 of 1042 events with zero-time duration between preceding usage session and Turning screen on were excluded) of Turning screen on events illustrated that 461 (49\%) of these events encompass similar behavioral patterns. Notably, it is easier for users to revert to their original status when the preceding usage status is Screen Off $(64 \%)(\chi 2=21.835, \mathrm{p}<.01)$. 


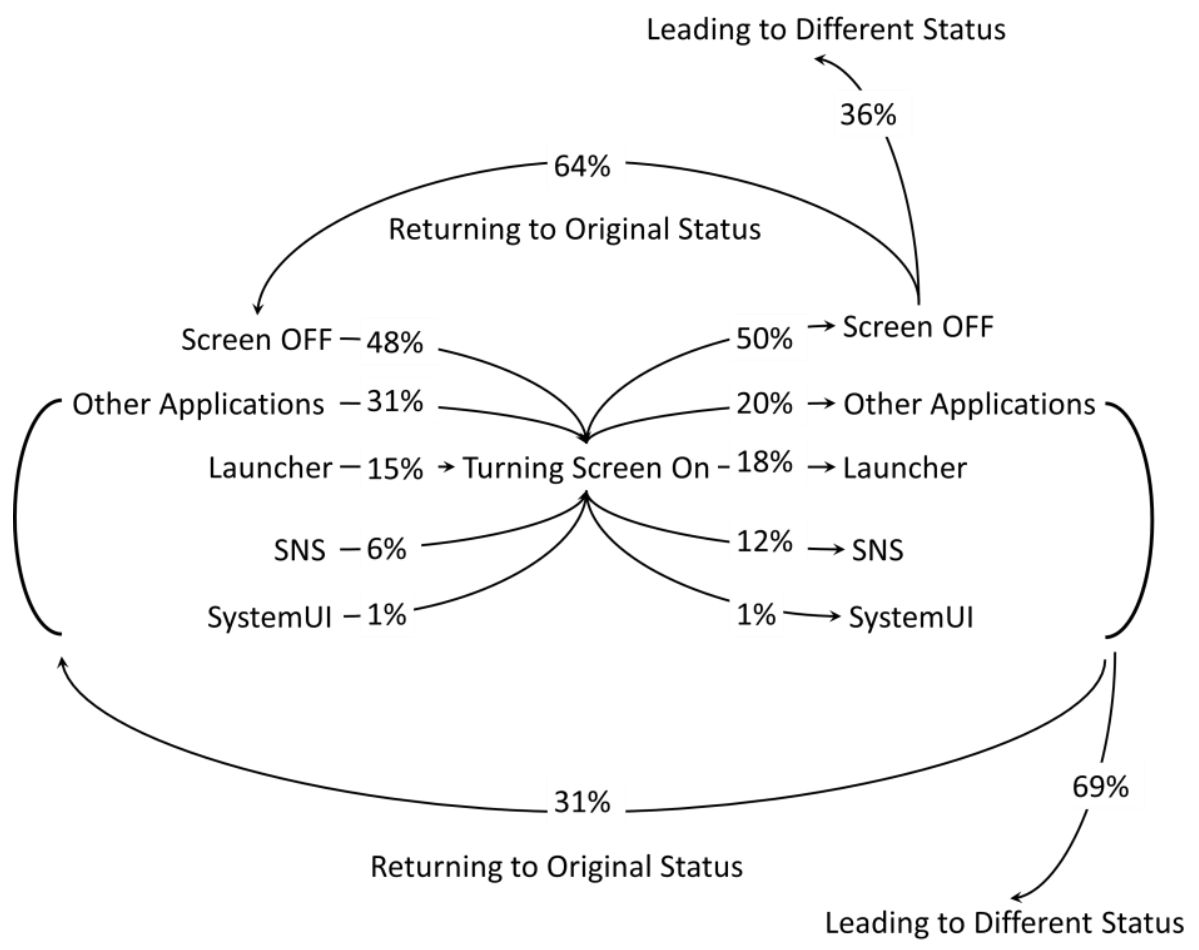

Fig. 1. User Behavior Related to Active Awareness

\subsection{Active Computer and Human Passive Awareness}

The level of surrounding light usually changes dynamically and an Auto-brightness feature on a mobile phone can sense the level of lighting in real-time and adjust the brightness of the screen both automatically and implicitly. Sensitivity to light events thus mirror the awareness capability of Auto-brightness feature to light events as illustrated in Fig. 3. Results indicate that the temporal distribution of light events (higher than 5000 Lux or lower than 200 Lux) and adjustment of screen brightness are highly correlated $(r=0.76)$. Nevertheless, the Sensitivity to light events of the Autobrightness feature is only $2 \%$ (Mean $=2 \%$; Max $=18 \%$; Min $=0$ ), thereby implying that the Auto-brightness feature, on average, only reacted to $2 \%$ of the light events specified in this study. The temporal distribution of Sensitivity to light events revealed that the Auto-brightness feature functions better in the morning and deteriorates drastically in performance at around 8 am when users began to utilize mobile applications intensively.

Results from the survey questionnaire show that users were generally satisfied with the Auto-brightness feature. Only six out of 24 participants ( 1 didn't utilize this feature 
and 7 didn't realize that this feature existed during the research) were somewhat or very dissatisfied with the Auto-brightness feature.

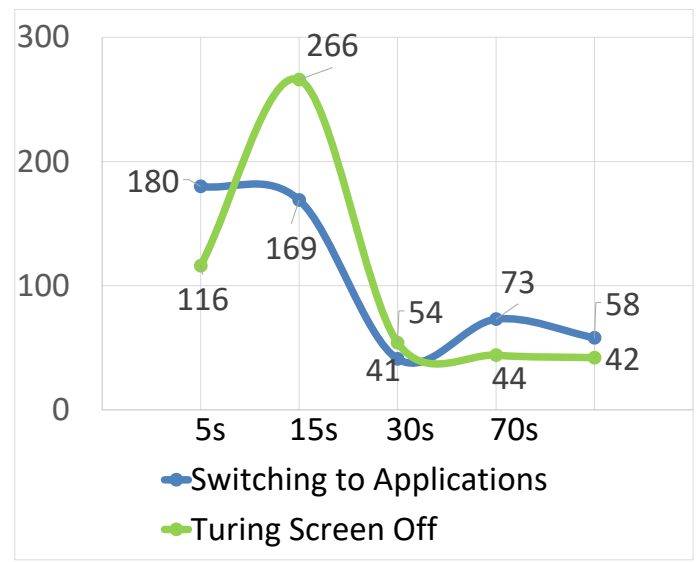

Fig. 2. Time Duration from Turning-screen-on to Follow-up Usage Session

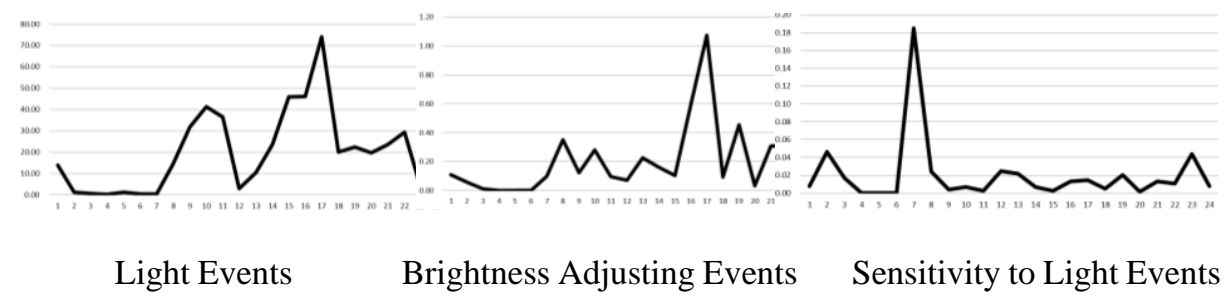

Fig. 3. Temporal distribution of Contextual Events

\subsection{Formation of UX}

Services which feature context-aware properties are ubiquitous in contemporary mobile applications. We reviewed the context-aware services running on our participants' Android mobile phones and found a total of 30 context-aware services that were incorporated into the mobile applications housed on participants' smart phones (see section 2.3 for details). In Fig. 4, we present the ratings of those 30 context-aware services that were utilized by more than half of our participant sample. Results indicate that mobile applications, which embody services exhibiting awareness of personal and social contexts (e.g., browsing habits, hobbies, location, shopping history, and social network) were least preferred. Conversely, mobile applications, which exhibit awareness of computing and physical contexts (e.g., internet connection, system language, and time zone) were most preferred. Taken together, these results suggest that users' preference for context-aware services are governed by the type(s) of context afforded through a mobile application. Users would like to retain control over the 
awareness of personalized contexts while at the same time, permitting smart phones to sense impersonal contexts and undertake suitable actions automatically.
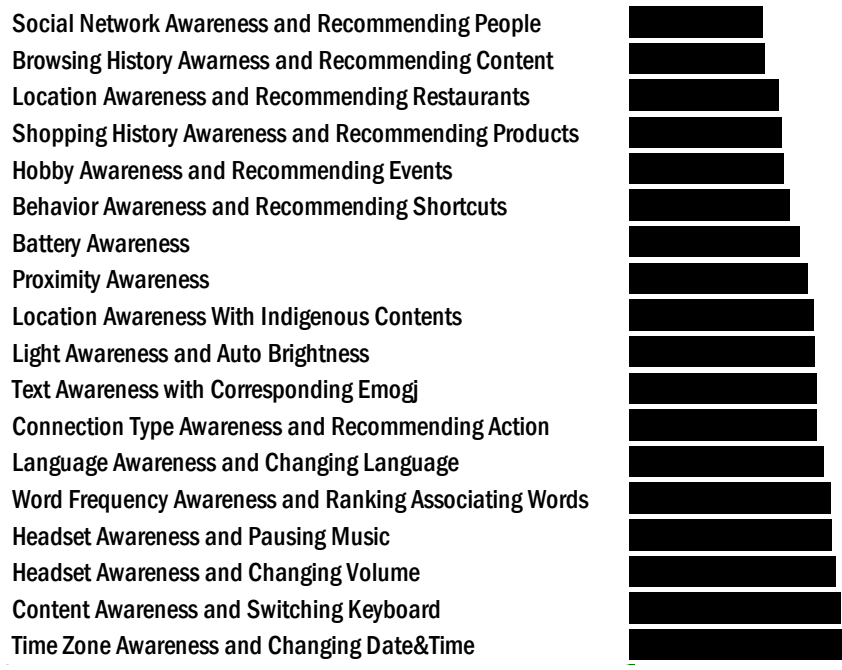

Fig. 4. Ratings of most common context-aware services found on participants' phones .

Open Coding of survey responses yielded 23 codes. Additionally, 5 categories were further identified through Axial Coding by scutinizing major UX concerns, conditions for producing UX concerns, actions/interactions of users and context-aware services produced in response to the UX concerns as well as their ensuing consequences. A core finding is that the UX of mobile context-aware applications, which includes instrumental meaning and value, was indeed formed from the interplay between smart phones' and users' awareness. Moreover, users also expressed frustration with the implementation and mode of interaction for certain mobile context-aware applications. This is exemplified through our conceptual model in Fig. 5 and excerpts from interviews as shown below.

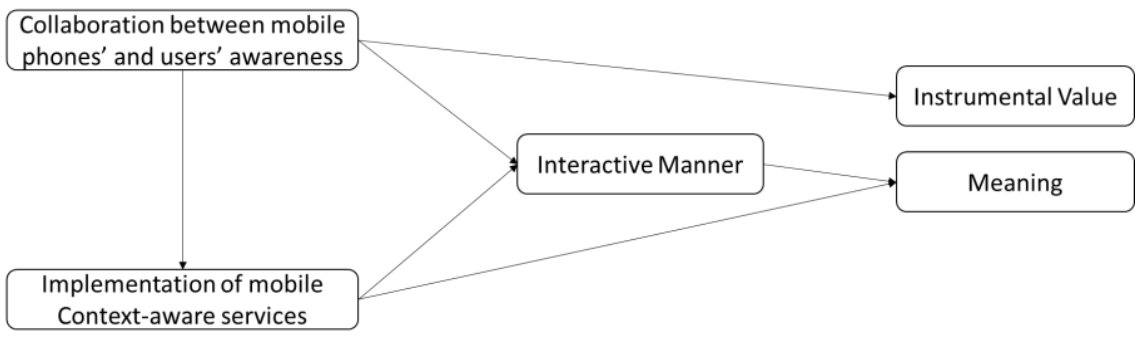

Fig. 5. Framework of UX constructs.

Collaboration between Smart Phones' and Users' Awareness: Consistent with our proposition that complementarities between smart phones' and users' awareness is 
pivotal in shaping the UX of mobile context-aware applications, we discovered that while smart phones' awareness is indeed capable of supplementing human ineptitude in allocating attention to changing contexts, it might impair human awareness under certain circumstances by cultivating an over-reliance on mobile devices. Additionally, we found that involving users in the interaction, catering for manual corrections in the event of malfunctions in mobile context-aware applications, and offering visible feedback about the outcomes of context-aware services are also deemed to be crucial ingredients for ensuring the quality of UX. Notably, awareness of users' hobbies and personal values, as raised by one of the Danish participant, could be considered as an often overlooked aspect of user context when designing context-aware services.

[Allowing for manual correction by human] "Saving battery. But if I was outside and it dims suddenly it might affect my usage and I have to adjust it manually." [P26]

[Complementing human ineptitude] "It's normal that I forgot to adjust the brightness of screen or night mode, so reminding me to night mode at certain time can help push me to do that;" [P31]

[Visibility of executing status] "Intelligent and saving time. But sometimes I might not be aware of that it has already adapted to the current input type, so I changed it manually and later on realized that I have to change it to the correct one." [P26]

[Adaptation to personal value and hobby] "Primarily based on what friends on social media are attending, and to my own preferences... " [P8]

[User in the loop] "I'm not sure whether I used this functionality, it's probably that I didn't pay attention to whether the screen is locked when I'm in a call. ” [P21]

[Impairing human awareness] "I might miss some events if the volume of alarm clock to be muted when the context is quiet." [P31]

Awaretiquette: What distinguishes context-aware services from their traditional counterpart is that the former is capable of initiating interaction with proactive action based on an intricate understanding of users' contextual environment. But at the same time, the proactivity of context-aware services introduces new concerns that do not exist in their more traditional reactive counterparts. As such, the proactive actions initiated by context-aware services should be acceptable, conducive, and desirable to users, just like humans behaving in good manners when communicating with one another.

[Protecting privacy] “... it wants to know everything and it invades privacy that they can get, a little annoying for me personally.” [P15]

[Polite proactive awareness] "Avoid the rudeness and embarrassment of bursting into music in quiet situation.” [P31] 
[Interruptability] "It gets very invading in some kind because It's constantly asking what are you thinking... so that's where it gets, annoying or too much invades” [P15]

[Way of presenting information] "It's very rude (not friendly) to remind me with the words "please continue to download if you are upstart wealthy", it's uncomfortable. " [P26]

[Trust] "Usually the sequence of recommending stores is based on the amount of expense of each store on ads, so I don't trust the recommending ones. I tend to trust what I searched by myself, it seems that this kind of recommendations are ads to me. I do $t$ want my phone to keep track on my behavior. It can be abused by third parties." [P31]

Implementation of Context-Aware Services: If active awareness were to be activated, mobile context-awareness applications should be accurate, intelligent, and sensitive in its execution. Mobile context-aware applications should not only allow related tasks progress smoothly without disrupting or annoying users, but they also let users control the execution as and when they want to.

[Sensitivity] "But if I was outside and it dims suddenly it might affect my usage and I have to adjust it manually." [P26]

[Accuracy], "The recommendations of Zhihu is really accurate.” [P21]

[Intelligence] “.... mobile context-aware systems and services service] enables a lot of the smart functionality is that when you walk, close to a metro station it will give you, the next departure times something even though i don't use it. So all of that isn't able by like the location right like using google maps as well location data." [P6]

[Controllability] "I'm not actually pretty sure because maybe I will feel like a little lose control. If, maybe at eleven o'clock is turned the light more down because sometimes I might not want it because sometimes I might be out, in the weekend, so, I'm not pretty sure actually what I want." [P9]

[Innovation] "I find the app very innovation but at the same time a bit unnecessary. Some of the things are very smart but some other not very necessary for me." [P9]

Instrumental Value: Not surprisingly, the quality of UX is associated with efficiency that stems from reduction in operations. Nevertheless, participants' feedback also indicates that the value of mobile context-aware applications might be discounted if it fails to demonstrate to users how it operates.

[Efficiency] "It help reduce the operations to switch between keyboards" [P27] 
[Intelligibility] "Not satisfied. I have no idea of how to do anything. I would like a better interface” [P8]

[Utility] "It's smart, can't image how it will be used; It did not actually once remind me of anything, I guess the conditions were too specific, hence they actually never met." [P15]

Meaning. The value of mobile context-aware applications extend beyond saving time and effort. Such applications can also bring about financial, health, and even social benefits to users.

[Health Value] "To protect eyes and healthy when staring at the phone screen." [P28]

[Financial benefit] "It's good that it can help save data." [P26]

[Social benefit] "Avoiding the rudeness and embarrassment of bursting into music in quiet situation" [P31]

\section{Discussion}

\subsection{A 'Hybrid Awareness' experience?}

This study sought to illustrate the interplay between smart phones' and users' awareness by examining the UX of 30 distinct context-aware services. We expect that UX concerns about mobile context-aware applications would not only differ from those of mobile applications in general, but they would also be rooted in the interplay between smart phones' and users' awareness.

Consistent with our predictions, we observed a discrete mode of usage behavior initiated by the interplay between smart phones' and users' awareness, as well as by potential challenges to user awareness that resulted from malfunctions of mobile context-aware applications. We further uncovered that the UX concerns of mobile context-aware applications were mainly associated with the interplay between contextaware services and users' awareness. Analytical results illustrated that the interplay between smart phones' and users' awareness shaped the mode of usage and that users possessed a high level of awareness about the contexts which mediate their usage of smart phones. Data of mobile applications with active awareness (implicit interaction) and passive awareness (explicit interaction) reveals that: (1) human awareness cannot be excluded from the loop of interaction in active awarness of mobile context-aware applications, and; (2) intelligibility/utility issues were major concerns in passive mobile context-aware applications. Finally, responses to our survey questionnaire show that while users favor their own retention of personal contexts, they are willing to grant permission to mobile context-aware applications to deal with impersonal contexts. 
Together, the preceding analytical results give rise to a conceptual model depicting how smart phones' and user' awareness interact with each other to realize quality in the UX of mobile context-aware applications, as can be deduced from the following quote by participant P14:

"it is difficult for me to answer, because I have to be aware of what's happening on my mobile phone, when I'm using it $i$ have to take care of what's happening on my telephone when it's just lying in my pocket do i have to be aware someone's calling me, $i$ have to be aware what it's doing. So there are passive and actvie or positive use scenarios" [P14].

Awareness of smart phones and users, in combination, form an awareness entity. Consequently, mobile context-aware applications should take into consideration users' awareness in sensing contexts, predicting users' intention, and executing actions. Beyond computing, spatial, social, and temporal contexts, user awareness should also be treated as a type of context in the design of context-aware services. Indeed, only a handful of studies have investigated how smart phones can initiate interaction based on user contexts, e.g., [8]. Therefore, by demonstrating that user's awareness of using/not using mobile phone is context-dependent, we draw attention to the importance of detecting users' awareness of their contextual environments and of deploying awareness strategies which complement users' awareness. Moreover, users possessed different types of awareness which should be taken into account as well. A quote from one of the participants may shed light on the constituents of a hybrid awareness system:

"...I have to be aware of what's happening on my mobile phone. When I'm using it, I have to take care of what's happening on my telephone and when it's just lying in my pocket I do have to be aware someone's calling me, I have to be aware what it's doing. So there are passive and active or positive use scenarios..." [P14].

To conceptualize the variations in UX that originate from the interplay between smart phones' and users' awareness, we advance the Hybrid Awareness System (HAS) model in Fig. 6 below. 


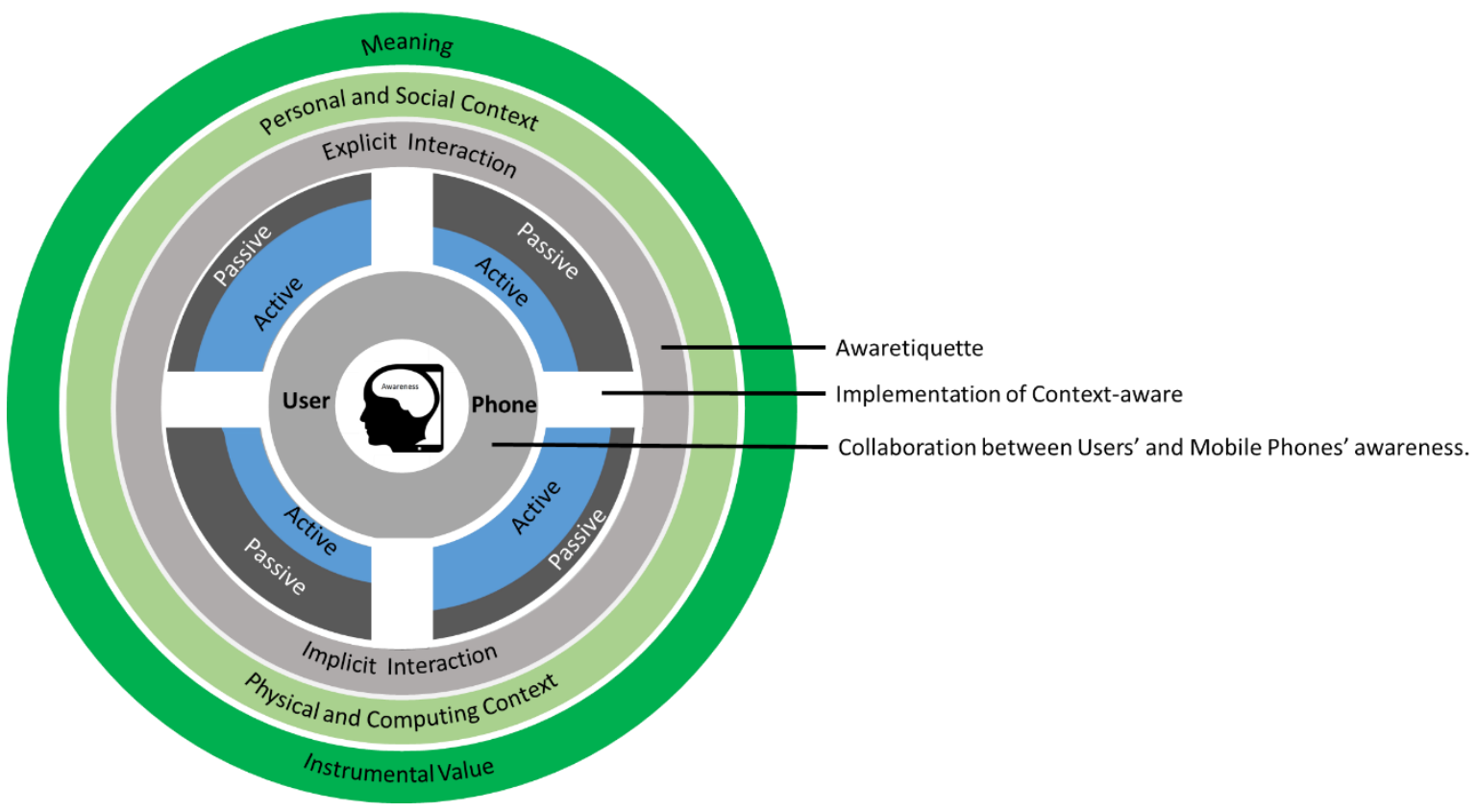

Fig. 6. Hybrid Awareness System (HAS) models the interplay between mobile phones' and users' active and passive awareness as dependent on the types of context. Varied forms of interaction are required to address UX concerns across distinct modes of interplay.

The conceptual model in Fig. 6. highlights four focal factors that govern the interplay between smart phones' and users' awareness. These four factors include awareness, interaction, context types, and UX. In this model, smart phones' and users' awareness constitute a hybrid awareness system in which the two types of awareness collaborate with each other in a dynamic fashion. When confronted with information on personal and social contexts, users' awareness takes precedence over that of smart phones in analyzing the information and taking appropriate actions. Conversely, when faced with information on computing and physical contexts, smart phones' awareness assumes the prominent role in interpreting the information before executing actions automatically and implicitly. Nonetheless, keeping users in the loop of interaction is still necessary even when the interaction is automatic and implicit.

\subsection{Implications for Design}

Context-aware services are ubiquitous thanks to the advances in computing capability and sensor technology of smart phones. Findings from this study indicate that the design of mobile context-aware applications should take smart phones' and users' awareness into account as a hybrid awareness entity. Compared with conventional ways of 
interaction featuring humans in proactive roles and computers in reactive roles, contextaware services are becoming more proactive, as they are able to detect contextual events and initiate interaction proactively based on their interpretation and understanding of human intention.

From the standpoint of human-computer interaction, the success of context-aware services is not only dependent on their efficiency and effectiveness in fullfilling delegated tasks (i.e., usability), it is also reliant on their: (a) comprehension of the state of users' awareness; (b) adoption of explicit or implicit interaction in accordance with the dynamic state of smart phones' and users' awareness, as well as; (c) prediction of user intention and initiation of interaction at opportune moments. Furthermore, the implementation of mobile context-aware applicattions should avoid impairing users' awareness by offering complementary solutions: designers should avoid scenarios whereby the performance of mobile context-aware applications deteriorate after users have become over-reliant on these applications.

Second, findings from this study call attention to the novel concept of what we label as 'awaretiquette' in the design of mobile context-aware applications. The design of mobile context-aware applications must not only cope with traditional issues of humancomputer interaction (e.g., privacy and trust), they should also deal with adverse effects attributed to proactive interactions from context-aware services. In Chinese culture, 'awaretiquette' refers to initiating appropriate interactive actions according to occasions (分场合) and to speaking separate languages to human and ghost (见人说人 话, 见鬼说鬼话). In contrast to mobile applications that are aware of physical contexts, those aware of social contexts are less desirable due to users' apprehension over losing control of social relationships. Consequently, designing socially-aware services need to pay attention to the issue of 'awaretiquette' by taking socio-cultural norms into consideration.

Finally, the design of mobile context-aware applications should consider the contribution of context-aware services to UX that goes beyond intrumental values (e.g., meaning to life). Undoubtably, automatic and implicit interactions afforded by contextaware services could lead to efficiency and low workload, thereby maximizing instrumental values. But at the same time, it may be accompanied by side effects such as users' being kept out of the loop, invisibility of system status, and relinquishing control. The design of mobile context-aware applications should hence exploit the strengths of context-aware services while concurrently, staying clear of their detrimental impact.

\subsection{Limitations and Future Work}

This study was carried out with a small sample size to explore the UX issues arising from the interplay between smart phones' and users' awareness. We derived the constructs and sub-dimensions of UX that is deserving of further inquiry in the future with a larger sample size.

Past studies have reported that humans easily generalize etiquettes for human-human interaction to human-computer interaction [34] so much so that computers are required to exhibit appropriate etiquette when interacting with humans. Although the etiquette 
issue has attracted a wealth of attention in the field of human-robot interaction [35-37], there is a dearth of research that has been devoted to the interaction between smart phones' and users' awareness [38]. As revealed in this study, smart phone users have equally experienced bad etiquette from mobile context-aware applications. Future research can concentrate on exploring the issue of etiquette associated with proactive and implicit interaction, especially pertaining to the disruption caused by switching among smart phones' and users' awareness.

In this study, we advanced a conceptual model based on interpreting findings relating to UX concerns as well as the interplay between smart phones' and users' awareness. The model calls for further and dedicated research with larger sample size to investigate specific issues such as the UX dimensions distinguishing context-aware services from context-unaware services as well as various modes of interplay between smart phones' and users' awareness for specific context-aware services.

Finally, a majority of participants in this study were students. While learning can be considered a type of work for students, future research should differentiate between students and employees, as well as between classrooms and workplaces, when considering the influence of the interplay between smart phones' and users' awareness on the UX of mobile context-aware applications.

\section{Conclusion}

In conclusion, our findings and proposed conceptual model support a dynamic view of UX that is shaped by the interplay between smart phones' and users' awareness. We attested to how the: (a) usage behavior of smart phone users are influenced by the interplay between smart phones' and users' awareness over time; (b) malfunction of mobile context-aware application might erode the UX by fostering over-reliance on smart phones' awareness, as well as; (c) four focal factors identified in this study govern the interplay between smart phones' and users' awareness. We advance a 'Hybrid Awareness System' (HAS) that models the interplay between smart phones' and users' active and passive awareness as dependent on the type of context. We allege that our novel term 'awaretiquette' may be leveraged to refer to initiating appropriate interactive actions according to occasions (分场合) and to speaking separate languages to human and ghost (见人说人话, 见鬼说鬼话).

\section{Acknowledgement}

This study was funded by the Marie Skłodowska-Curie Action, grant number 708122 , project Mobile context-aware cross-cultural applications (MOCCA). The log tools (Android) are at http//sf.cbs.dk/mocca or at https://www.researchgate.net/project/Mobile-context-aware-cross-culturalapplications-Funded-by-Marie-Sklodowska-Curieactions/update/5bb0ccab3843b006753be80c?_iep1\%5BviewId\%5D=cxK4iY1Jj37HgJ 
qqYGZbB1KM\&_iepl\%5Bcontexts\%5D\%5B0\%5D=projectUpdatesLog\&_iepl\%5Bi nteractionType $\% 5 \mathrm{D}=$ projectUpdateDetailClickThrough.

\section{$7 \quad$ References}

1. The International Telecommunication Union (ITU): ICT Figures - The world in 2015. Geneva (2015).

2. Duke, É., Montag, C.: Smartphone addiction, daily interruptions and selfreported productivity. Addict. Behav. Reports. 6, 90-95 (2017).

3. Weber, D., Voit, A., Exler, A., Schröder, S., Böhmer, M., Okoshi, T.: Intelligent notification and attention management on mobile devices. Proc. 16th Int. Conf. Mob. Ubiquitous Multimed. 561-565 (2017).

4. Weber, D., Shirazi, A.S., Gehring, S., Henze, N., Poppinga, B., Pielot, M., Okoshi, T.: Smarttention, Please!: 2nd Workshop on Intelligent Attention Management on Mobile Devices. In: Proceedings of the 18th International Conference on Human-Computer Interaction with Mobile Devices and Services Adjunct. International Conference on Human-Computer Interaction with Mobile Devices and Services (MobileHCI), September 6-9, Florenz, Italy. pp. 914-917 (2016).

5. Paul, C.L., Komlodi, A., Lutters, W.: Interruptive notifications in support of task management. Int. J. Hum. Comput. Stud. 79, 20-34 (2015).

6. Okoshi, T., Nozaki, H., Nakazawa, J., Tokuda, H., Ramos, J., Dey, A.K.: Towards attention-aware adaptive notification on smart phones. Pervasive Mob. Comput. 26, 17-34 (2016).

7. Oulasvirta, A., Rattenbury, T., Ma, L., Raita, E.: Habits make smartphone use more pervasive. Pers. Ubiquitous Comput. 16, 105-114 (2012).

8. Pielot, M., Rello, L.: The do not disturb challenge: A day without notifications. In: Proceedings of the 33rd Annual ACM Conference Extended Abstracts on Human Factors in Computing Systems. pp. 1761-1766. ACM (2015).

9. Barkhuus, L., Dey, A.: Is context-aware computing taking control away from the user? three levels of interactivity examined. In: International Conference on Ubiquitous Computing. pp. 149-156. Springer, Berlin, Heidelberg (2003).

10. Bellotti, V., Edwards, K.: Intelligibility and accountability: Human considerations in context-aware systems. Human-Computer Interact. 16, 193212 (2001).

11. Evers, C., Kniewel, R., Geihs, K., Schmidt, L.: The user in the loop: Enabling user participation for self-adaptive applications. Futur. Gener. Comput. Syst. 34, 110-123 (2014).

12. Temdee, P., Prasad, R.: Context-aware communication and computing: applications for smart environment. Springer, Switzerland (2017).

13. Kaber, D.B., Endsley, M.R.: The effects of level of automation and adaptive automation on human performance, situation awareness and workload in a dynamic control task. Theor. Issues Ergon. Sci. 5, 113-153 (2004).

14. Endsley, M.R.: Automation and situation awareness. autom. hum. perform. 
Theory Appl. 163-181 (1996).

15. Whitaker, R.M., Chorley, M., Allen, S.M.: New frontiers for crowdsourcing: The extended mind. Proc. Annu. Hawaii Int. Conf. Syst. Sci. 2015-March, 1635-1644 (2015).

16. Katre, D.: One-handed thumb use on smart phones by semi-literate and illiterate users in India. In: Katre, D., Orngreen, R., Yammiyavar, P., and Clemmensen, T. (eds.) HWID 2009 - Human Work Interaction Design: Usability in Social, Cultural and Organizational Contexts. IFIP Advances in Information and Communication Technology. pp. 189-208. Springer, Berlin, Heidelberg (2010).

17. Wurhofer, D., Fuchsberger, V., Meneweger, T., Moser, C., Tscheligi, M.: Insights from user experience research in the factory: What to consider in interaction design. In: Nocera, J.A., Barricelli, B., Lopes, A., Campos, P., and Clemmensen, T. (eds.) HWID2015 -Human Work Interaction Design. Work Analysis and Interaction Design Methods for Pervasive and Smart Workplaces. IFIP Advances in Information and Communication Technology. pp. 39-56. Springer, Berlin, Heidelberg (2015).

18. Yammiyavar, P., Kate, P.: Developing a mobile phone based GUI for users in the construction industry: A case study. In: Katre, D., Orngreen, R., Yammiyavar, P., and Clemmensen, T. (eds.) HWID 2009 - Human Work Interaction Design: Usability in Social, Cultural and Organizational Contexts. pp. 211-223. Springer, Berlin, Heidelberg (2010).

19. López, G., Guerrero, L.A.: Ubiquitous notification mechanism to provide user awareness. In: Advances in Ergonomics in Design. pp. 689-700. Springer International Publishing (2018).

20. Bakker, S., Niemantsverdriet, K.: The interaction-attention continuum: Considering various levels of human attention in interaction design. Int. J. Des. 10, 1-14 (2016).

21. Dey, A.K., Häkkilä, U.J.: Context-awareness and mobile devices. In: Handbook of research on user interface design and evaluation for mobile technology. pp. 205-217. IGI Global (2008).

22. Pradhan, S., Qiu, L., Parate, A., Kim, K.H.: Understanding and managing notifications. Proc. - IEEE INFOCOM. 1-9 (2017).

23. Schneegass, S., Rzayev, R.: Embodied notifications: Implicit notifications through electrical muscle stimulation. Proc. 18th Int. Conf. Human-Computer Interact. with Mob. Devices Serv. Adjun. 954-959 (2016).

24. Okoshi, T., Ramos, J., Nozaki, H., Nakazawa, J., Dey, A.K., Tokuda, H.: Attelia: Reducing user's cognitive load due to interruptive notifications on smart phones. 2015 IEEE Int. Conf. Pervasive Comput. Commun. PerCom 2015. 96-104 (2015).

25. Voit, A., Henze, N., Poppinga, B., Gehring, S., Weber, D., Okoshi, T., Böhmer, M., Pejovic, V.: UbiTtention: Smart \& ambient notification and attention management - Workshop in conjunction with UbiComp 2016. 1520-1523 (2016).

26. Iqbal, S.T., Horvitz, E.: Notifications and awareness: A field study of alert 
usage and preferences. In: Proceedings of the 2010 ACM conference on Computer supported cooperative work. pp. 27-30. ACM (2010).

27. Lee, Y.K., Chang, C.T., Lin, Y., Cheng, Z.H.: The dark side of smartphone usage: Psychological traits, compulsive behavior and technostress. Comput. Human Behav. 31, 373-383 (2014).

28. Hong, J. yi, Suh, E. ho, Kim, S.J.: Context-aware systems: A literature review and classification. Expert Syst. Appl. 36, 8509-8522 (2009).

29. Kwon, O., Choi, K., Kim, M.: User acceptance of context-aware services: Selfefficacy, user innovativeness and perceived sensitivity on contextual pressure. Behav. Inf. Technol. 26, 483-498 (2007).

30. Qin, X., Tan, C.W., Bødker, M., Sun, W., Clemmensen, T.: Culturally informed notions of mobile context awareness - Lessons learned from user-centred exploration of concepts of context and context awareness. In: IFIP Conference on Human-Computer Interaction. Lecture Notes in Computer Science (including subseries Lecture Notes in Artificial Intelligence and Lecture Notes in Bioinformatics). pp. 420-440 (2017).

31. Qin, X., Tan, C.W., Clemmensen, T.: Context-awareness and mobile HCI: Implications, challenges and opportunities. In: Lecture Notes in Computer Science (including subseries Lecture Notes in Artificial Intelligence and Lecture Notes in Bioinformatics) (2017).

32. Johnson, R.B., Onwuegbuzie, A.J., Turner, L.A.: Toward a definition of mixed methods research. J. Mix. Methods Res. 1, 112-133 (2007).

33. Adams, A., Lunt, P., Cairns, P.: A qualititative approach to HCI research. In: Cairns, P. and Cox, A.L. (eds.) Research Methods for Human Computer Interaction. pp. 138-157. Cambridge University Press, Cambridge (2008).

34. Reeves, B., Nass, C.: The media equation: How people treat computers, television, and new media like real people and places. Cambridge University Press (1996).

35. Dautenhahn, K.: Socially intelligent robots: Dimensions of human-robot interaction. In: Philosophical Transactions of the Royal Society B: Biological Sciences. pp. 679-704 (2007).

36. Kidd, C.D., Breazeal, C.: Robots at home: Understanding long-term humanrobot interaction. In: 2008 IEEE/RSJ International Conference on Intelligent Robots and Systems, IROS. pp. 3230-3235 (2008).

37. Saerbeck, M., Schut, T., Bartneck, C., Janse, M.D.: Expressive robots in education: Varying the degree of social supportive behavior of a robotic tutor. Proc. 28th Int. Conf. Hum. factors Comput. Syst. - CHI '10. 1613-1622 (2010).

38. Cheverst, K., Davies, N., Mitchell, K., Friday, A., Efstratiou, C.: Developing a context-aware electronic tourist guide. In: Proceedings of the SIGCHI conference on Human factors in computing systems - CHI '00. pp. 17-24 (2000) 